\title{
Investigação bibliográfica e análise do potencial de dispersão de sementes por aves frugívoras no Brasil
}

\author{
Ana Carolina Ferreira', Ariodari Francisco dos Santos $^{2} \&$ Huilquer Francisco Vogel ${ }^{3}$ \\ 'Graduada em Ciências Biológicas pela Universidade Estadual do Centro Oeste, Campus CEDETEG, Guarapua- \\ va-PR.Email: nina.bio2@gmail.com \\ ${ }^{2}$ Departamento de Ciências Biológicas da Universidade Estadual do Centro Oeste, Campus CEDETEG, Guara- \\ puava-PR. Email: ariodari@unicentro.br \\ ${ }^{3}$ Centro de Ciências Exatas e Biológicas da Universidade Estadual do Paraná, Campus União da Vitória, União \\ da Vitória-PR.Email: huilquer@hotmail.com

\begin{abstract}
Bibliographical research and analysis of seed dispersal potential by frugivorous birds in Brazil. Birds have great participation in the dispersion process, although sometimes the effect can be negative. The aim of this study was to determine a group containing species of birds, which can be found in different biomes of Brazil and can be considered as effective dispersers. We conducted a literature review of studies on frugivory and developed an array of data relating bird species and species of plants. With the assistance of a multivariate discriminant analysis based on 34 articles between the years 1990 and 2010, it was possible to isolate 14 species with extremely favorable dispersion, being considered as effective dispersers. Among these, it was highlighted Turdus rufiventris, Turdus leucomelas, Turdus amaurochalinus and Tangara sayaca that, besides having the main features, have a high frequency of occurrence, contributing to the quality of the dispersion.
\end{abstract}

Key words: Feeding behavior, Frugivory, Ornitochory.

Resumo. Aves exercem grande participação no processo de dispersão, embora em algumas vezes o efeito possa ser negativo. O objetivo deste trabalho foi determinar quais espécies de aves no Brasil podem ser consideradas efetivas dispersoras. Para tanto, foi realizado um levantamento bibliográfico de estudos sobre frugivoria e elaborada uma matriz de dados relacionando espécies de aves e de plantas. Com o auxílio de uma análise discriminante multivariada baseada em 34 artigos entre os anos de 1990 e 2010, foi possível isolar 14 espécies com características extremamente favoráveis à dispersão, sendo consideradas dispersoras efetivas. Destas, destacam-se Turdus rufiventris, Turdus leucomelas, Turdus amaurochalinus e Tangara sayaca que, além de possuírem as principais características favoráveis à dispersão, apresentam uma alta frequência de ocorrência, contribuindo ainda mais para a qualidade da dispersão.

Palavras-chave: Comportamento alimentar, Frugivoria, Ornitocoria. 


\section{INTRODUÇÃO}

Devido à falta de mobilidade das plantas, seu sucesso reprodutivo é dependente de mecanismos de dispersão através da água, vento ou por interdependência com animais e insetos polinizadores (RUDGE, 2008). De acordo com ANTUNES \& RIBEIRO (1999), o sucesso reprodutivo de algumas espécies vegetais é extremamente dependente da sua interação com a avifauna dispersora. Quando as aves consomem frutos, as sementes são carregadas durante o voo passando pelo trato digestivo ou são regurgitadas, disseminando-se por uma ampla variedade de habitats (GONDIM, 2001).

Alguns fatores comportamentais das aves frugívoras podem afetar o sucesso reprodutivo da planta, caracterizando a ave como boa ou má dispersora. Neste aspecto, o modo de apanhar e de manipular os frutos no bico, frequência de visitas, quantidade de frutos ingeridos em cada visita (HERRERA \& JORDANO, 1981), preferências alimentares (FAUSTINO \& MACHADO, 2006) e também a qualidade e distância da deposição das sementes no ambiente podem ser fatores limitantes.

Segundo Albuquerque et al., (2006), a eliminação de excretas destas aves sobre áreas perturbadas desempenha papel fundamental no processo de regeneração florestal, contribuindo de forma natural para a disseminação de propágulos, o que favorece a conservação de ecossistemas. Ainda, a capacidade das aves de disseminarem sementes para longas distâncias, propicia a colonização de novas áreas e auxilia na regeneração de locais impactados (ANTONINI, 2007).

Entretanto, conforme estudos de SANTOS \& TELLERIA (1994), áreas fragmentadas tendem a possuir baixa riqueza de agentes dispersores, logo, a existência de plantas dependentes destas espécies também é reduzida, ficando sujeitas a perda de variabilidade genética, efeito de borda e crescimento na população de espécies pioneiras e invasoras (KAGEYAMA et al., 1998; BORGES et al., 2004;).

No Brasil, muitos estudos enfatizam a importância da interação ave/planta, especialmente em ambientes degradados. RABELLO et al., (2010), por exemplo, observaram que fragmentos florestais maiores tinham relação com o aumento de interação entre Copaifera langsdorffi e seus dispersores efetivos, gerando efeitos positivos tanto para a planta, quanto para as aves. FrANCISCO \& GALETTI (2002) verificaram a importância da avifauna dispersora de frutos de Ocotea pulchella em áreas degradadas, inclusive o papel fundamental exercido pelas espécies generalistas na escassez de frugívoros específicos neste ambiente.

Buscar compreender a dinâmica das aves dispersoras na natureza, bem como a sua atuação no processo de recuperação ambiental é de grande valia para incorporação de projetos de conservação. Diante disso, este trabalho objetiva diagnosticar com base em estudos já desenvolvidos no Brasil, quais grupos de aves possuem as características mais eficientes no processo de 
dispersão para que, desta forma, possam ser direcionadas estratégias de manejo de espécies e do habitat.

\section{Material e Métodos}

Inicialmente, foi realizado um levantamento bibliográfico utilizando como palavras-chave os termos "ornitocoria", "aves frugívoras", "comportamento alimentar", "dispersão de sementes" e "frugivoria por aves". Dessa forma, foram encontrados 98 resultados, que passaram por análise com base em critérios pré-estipulados. Artigos que compreendiam pesquisas com mamíferos ou que fossem fora do território brasileiro foram prontamente descartados. O critério de inclusão foi a observação de uma única espécie vegetal com a listagem das aves visitantes em determinado período. Desta forma, seria possível amostrar uma maior quantidade de espécies de aves, utilizando-se de um menor número de espécies vegetais, evitando desviar do foco da pesquisa. Aplicando este filtro de resultados, foram mantidos 34 artigos publicados entre $1990 \mathrm{e}$ 2010 para a realização das análises.

Assim, foi elaborada uma matriz de presença e ausência, relacionando cada espécie de ave com a planta da qual se alimentam. Ainda, em relação à frequência de ocorrência, utilizando o índice $C=p \times 100 / N$ de acordo com Dajoz (1983), onde $p$ é o número de ocorrência da espécie analisada e $N$ o número total de plantas listadas, foram propostas as seguintes categorias de agrupamento: Constante $=>50 \%$; Acessória $=$ $25-50 \%$ e Acidental $=<25 \%$.
A partir destes dados, cada espécie de ave foi incluída em categorias relativas a habitat, dieta e modo de manipulação do fruto (Apêndice B). Em relação ao habitat, as espécies foram categorizadas de acordo com a sua dependência florestal, baseando-se em escritos de STOTz et al., (1996), Silva et al., (2003) e ARAújo et al., (2012). Desta forma, puderam ser organizadas em dependentes (DEP), semidependentes (SMD) e independentes (IND). A dieta relacionada a cada espécie foi baseada, principalmente, nos relatos de SICK (1997) e WILLIS (1979), distribuindo-as em onívoras (ONI), insetívoras (INS), frugívoras (FRU) e granívoras (GRA). O modo de manipulação do fruto teve como referência os trabalhos de LEVEY (1987), MANHÃES (2003), GONDIM (2001) e FRANCISCO \& GALETTI (2002), diferenciando as espécies pela principal forma de ingestão do fruto. Assim, foram classificadas como engolidoras de fruto inteiro (EFI), mandibuladoras (MEF) e predadoras de semente (PS). Características do ambiente, tais como fragmentação e grau de perturbação não foram consideradas, mantendo o foco somente nos hábitos naturais das espécies de aves.

Para a plotagem dos dados cada categoria recebeu pesos variando de -2 a 2,5, em ordem crescente de importância, considerando uma pontuação mais alta para as características principais que o efetivo dispersor deve ou não apresentar e mais baixas para as características que prejudicam a dispersão. Algumas características não obtiveram nenhum valor por não representarem necessariamente um ponto relevante no processo de dispersão. Neste caso, incluímos 
a categoria dos mandibuladores, em que podem ou não favorecer a dispersão. Também os granívoros, que podem não inviabilizar a semente de acordo com o seu modo de consumo. Logo, à categoria dos insetívoros foi atribuída uma pontuação negativa por não incluírem sementes em sua dieta predominante. Valores negativos também foram atribuídos aos predadores de sementes, que normalmente destroem as sementes e prejudicam a dispersão. Assim, foram criadas espécies hipotéticas (E1, E2, E3, I1, 12, 13) para serem consideradas como grupo controle, com os valores máximos e mínimos esperados em cada categoria (Tabela 1). da somatória dos valores agregados, esperava-se destacar os efetivos dispersores dentre aqueles que obtivessem pontuação entre 9,3 e 6,8. Dispersores ineficientes foram considerados entre os valores de -2 e 1,5. Entretanto, para fins de análise, tanto estes quanto aqueles que atingiram valores intermediários (de 1,51 a 6,79) foram considerados dispersores ocasionais.

\section{Utilizando o software PAST - Palaento-} logical Statistics, através de uma análise discriminante multivariada, as espécies foram divididas em dois grupos, enquadrando-as de acordo com os valores pré-estabelecidos para as espécies hipotéticas. A análise discriminante utilizada é

Tabela 1. Demonstração dos valores utilizados para a plotagem de dados.

\begin{tabular}{|c|c|c|c|c|c|c|}
\hline \multirow{2}{*}{ Parâmetros } & \multicolumn{3}{|c|}{ Dispersores efetivos } & \multicolumn{3}{|c|}{ Dispersores ineficientes } \\
\hline & E1 & E2 & E3 & I1 & 12 & 13 \\
\hline \multicolumn{7}{|l|}{ HABITAT } \\
\hline Dependentes & 1 & 1 & 1 & 1 & 1 & 1 \\
\hline Semidependentes & 1,1 & 1,1 & 1,1 & 0 & 0 & 0 \\
\hline Independentes & 1,2 & 1,2 & 1,2 & 0 & 0 & 0 \\
\hline \multicolumn{7}{|l|}{ DIETA } \\
\hline Frugívoro & 2,5 & 2,5 & 0 & 2,5 & 0 & 0 \\
\hline Insetívoro & 0 & 0 & 0 & 0 & -1 & -1 \\
\hline Onívoro & 2 & 0 & 2 & 0 & 2 & 0 \\
\hline \multicolumn{7}{|l|}{ MANIPULAÇÃO } \\
\hline Engole fruto inteiro & 1,5 & 1,5 & 1,5 & 0 & 0 & 0 \\
\hline Preda semente & 0 & 0 & 0 & -2 & 0 & -2 \\
\hline Total & 9,3 & 7,3 & 6,8 & 1,5 & 2 & -2 \\
\hline
\end{tabular}

$E=$ efetivo; I = ineficiente 
em trabalhos que visem a separação de grupos em duas ou mais classes previamente definidas (JOHNSON \& WICHERN, 1988). Os resultados desta análise foram cruzados com a frequência de ocorrência e distribuídos em um gráfico de dispersão para posterior análise. A confecção do mapa e gráficos foi feita com o auxílio dos softwares GraphPad Prism ${ }^{\circledR}$ e Quantum GIS.

\section{Resultados e Discussão}

Os artigos selecionados apresentaram dados compreendendo os estados da região Sul e Sudeste, além do Pará e Mato Grosso, envolvendo 50 espécimes vegetais e 193 espécies de aves divididas em 33 famílias (CBRO, 2013). As mais ricas, em ordem decrescente, foram Thrau- pidae (38 espécies), Tyrannidae (30 espécies) e Psittacidae (13 espécies). Após a análise, 14 espécies (7\%) foram caracterizadas como efetivas dispersoras, citadas em ordem decrescente de acordo com a frequência de ocorrência registrada: Tangara sayaca, Turdus rufiventris, Turdus leucomelas, Turdus amaurochalinus, Pitangus sulphuratus, Empidonomus varius, Euphonia chlorotica, Tangara palmarum, Turdus flavipes, Manacus manacus, Tangara cayana, Penelope obscura, Ramphastos toco e Ortalis canicollis (Figura 1).

Em relação às guildas alimentares registradas, não houve dominância efetiva, ocorrendo um equilíbrio entre os hábitos de onivoria (36\%),

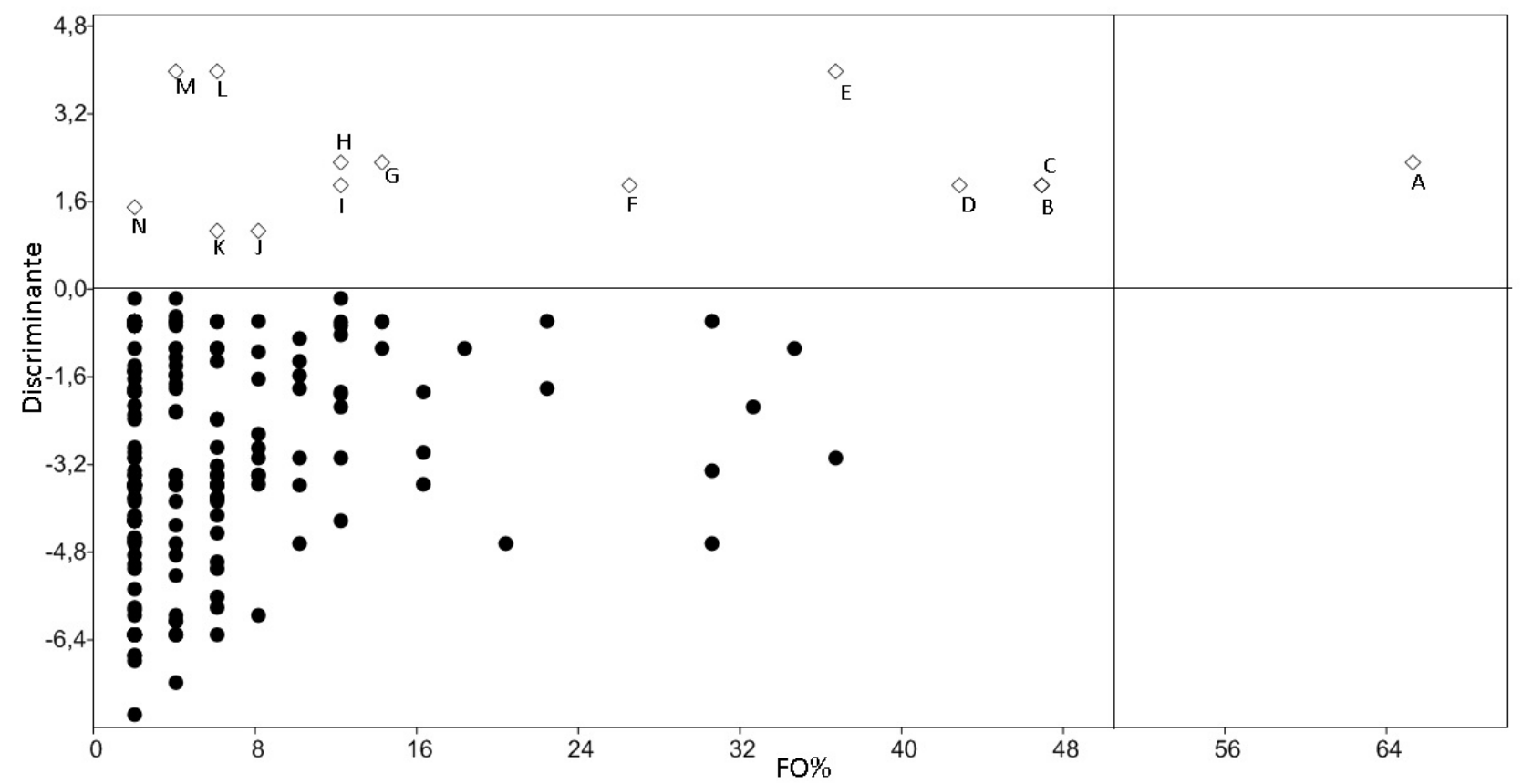

Figura 1. Análise discriminante demonstrando a separação dos grupos entre efetivos dispersores ( ) e dispersores ocasionais ( ). A) Tangara sayaca, FO =65\%; B) Turdus rufiventris, FO = 47\%; C) Turdus leucomelas, FO = 47\%; D) Turdus amaurochalinus, FO =43\%; E) Pitangus sulphuratus, FO = 37\%; F) Empidonomus varius, FO = 26\%; G) Euphonia chlorotica, FO = 14\%; H) Tangara palmarum, FO = 12\%; I) Turdus flavipes, FO = 12\%; J) Manacus manacus, FO = 8\%; K) Tangara cayana, $\mathrm{FO}=33 \%$; L) Penelope obscura, $\mathrm{FO}=6 \%$; M) Ramphastos toco, $\mathrm{FO}=4 \%$; $\mathrm{N}$ ) e Ortalis canicollis, $\mathrm{FO}=2 \%$. $* \mathrm{FO}$ : Frequência de Ocorrência. 
frugivoria (35\%) e insetivoria (34\%). Entretanto, ao relacionar estas características com o habitat, ocorre a predominância de espécies onívoras com preferência por áreas abertas e de frugívoros por ambientes florestais. Esta discrepância era esperada, uma vez que espécies frugívoras são frequentemente encontradas em maior número em áreas preservadas, e espécies de dieta generalista possuem melhor plasticidade à ambientes alterados (OLIVEIRA et al., 2009).

ARgel-DE-OliveirA (1998) salienta a importância da existência de características essenciais para a dispersão, tanto em aves quanto nas próprias sementes. Sendo assim, a dispersão efetiva só ocorre através da associação de vários fatores, com destaque para a influência da frequência de visitação, comportamento em relação ao ambiente onde se alimenta e a forma de ingestão.

Apesar de apresentarem as características favoráveis, $57 \%$ das espécies amostradas relacionadas como efetivas foram consideradas acidentais, o que afeta a qualidade da dispersão. Neste aspecto, destacam-se as famílias Turdidae, Thraupidae e Tyrannidae, com maior frequência e predominância nas visitas a árvores frutíferas. Destas, Tangara sayaca foi a única espécie considerada constante, com frequência de $65 \%$. A capacidade de ocuparem habitats distintos é de grande importância, sendo relevantes no processo de regeneração de áreas degradadas (MARCONDES-MACHADO \& ROSA, 2005). De acordo com MANHÃES et al., (2003), espécies como Penelope superciliaris e Lanio melanops deposi- tam sementes no interior da mata, colaborando para o crescimento das plântulas de maneira tardia, desenvolvendo-se quando ocorrer a formação de clareiras. Espécies de dieta generalista e que habitam áreas já impactadas, são capazes de percorrer locais abertos entre os fragmentos florestais, disseminando os propágulos de maneira mais homogênea e em áreas com maior necessidade de regeneração, promovendo uma colonização em curto prazo.

O modo de manipulação do fruto para ingestão também constitui um processo fundamental na dispersão. Para Sıck (1997), aves predadoras de sementes ou que mandibulam, fragmentando o fruto antes de engoli-lo, são consideradas más dispersoras. Em contrapartida, de acordo com MARCONDES-MACHADO (2002), os frutos engolidos inteiros teriam maior chance de sucesso, pois todas as sementes contidas nele seriam dispersas. Desta maneira, as sementes são carregadas para outros locais e, ao serem regurgitadas ou defecadas, saem intactas e com melhores condições para a germinação do que se permanecessem próximas à planta-mãe, onde a competição por recursos seria maior. Com base nestas características, $71 \%$ das espécies relacionadas apresentaram o hábito de engolir o fruto inteiro contra $23 \%$ que frequentemente mandibulam o fruto. Apenas $12 \%$ foram considerados predadores de sementes.

Embora frequentemente os indivíduos da família Thraupidae demonstrem o comportamento de fragmentar os frutos, de acordo com LEVEY (1987) e MANHÃES (2003) este hábito não 
impede que as sementes sejam efetivamente dispersas. Dependendo do tamanho do fruto, as sementes são normalmente engolidas junto com os pedaços do arilo, escapando da ação separadora do bico. Vários trabalhos citam a importância de Tangara sayaca como dispersor de diversas espécies de plantas, sem danificar suas sementes pelo hábito de mandibular e, muitas vezes, engolindo pequenos frutos inteiros (GONDIM, 2001; VAlente, 2001; CAZETTA et al., (2002).

A fragmentação florestal também é um dos fatores limitantes no processo de dispersão, afetando diretamente o potencial das aves dispersoras e o seu desempenho no auxílio da regeneração da mata, bem como a produção de frutos da população vegetal (NASCIMENTo et al., 1999; REIS et al., 1999; PIZO \& VIEIRA, 2004). Grande parte da avifauna que vive preferencialmente no interior da mata, são particularmente prejudicadas pela fragmentação, pois são incapazes de atravessar ambientes abertos, sentindo-se ameaçadas e expostas nestas áreas (PRIMACK \& ROdRIGUeS, 2001; JORdANO et al., 2006). Portanto, as espécies vegetais ornitocóricas são afetadas como consequência da sua dependência destes animais para a dispersão de sementes. As aves de grande porte, como Cracidae e Ramphastidae são um reflexo desta situação. Apesar de apresentarem elevado potencial de dispersão, tem sua frequência de ocorrência reduzida, devido, principalmente, à caça e à fragmentação florestal, afetando plantas que dependam exclusivamente destas espécies para sua dispersão (JORDANo et al., 2006). Os repre- sentantes da família Turdidae, por sua vez, estão amplamente distribuídos, tendo sua eficiência na dispersão corroborada em diversos trabalhos (GUERRERO; FIgUeIREDO, 1997; FrANCISCO; GALETTI, 2001; FONSECA; ANTUNES, 2007).

Outro fator preocupante é relacionado às aves frugívoras especialistas. Como possuem uma dieta bastante específica, a perda de habitat pode significar total restrição ao alcance dos seus recursos alimentícios, sendo suscetíveis à extinção. Ao contrário, aves generalistas podem se beneficiar desta diminuição de competição por recursos dentro de um mesmo fragmento. Logo, o tamanho do fragmento influencia a presença e diversidade de dispersores adequados, sendo prejudicial nas porções de mata com baixa extensão (MUSTAJARVI, 2001).

\section{CONCLUSÃo}

Com base nos dados coletados, foi constatado que em torno de 14 espécies podem ser consideradas efetivas dispersoras, onde Tangara sayaca é classificada como constante. As famílias Turdidae, Thraupidae e Tyrannidae apresentaram-se como as mais favoráveis neste sentido. Deste modo, novos estudos podem ser direcionados avaliando a viabilidade e qualidade da dispersão proporcionada por estes grupos.

\section{REFERÊNCIAS BIBLIOGRÁFICAS}

Albuquerque, L. B.; Velázquez, A. \& Mayorga-SAUCEDo, R. 2006. Solanaceae composition, pollination and seed dispersal syn- 
8. FERREIRA et al.

dromes in mexican mountain cloud Forest. Acta Botanica Brasilica, Feira de Santana, (20): 599-614.

ANTONINI, R. D. 2007. Frugivoria e dispersão de sementes por aves em duas espécies de Miconia (Melastomataceae) em uma área de Mata Atlântica na Ilha de Marambaia, RJ. Dissertação (Mestrado em Biologia Animal). Universidade Federal Rural do Rio de Janeiro, Seropédica.

Antunes, N. B. \& RibeiRo, J. F. 1999. Aspectos fenológicos de seis espécies vegetais em Matas de galeria do Distrito Federal. Pesquisa Agropecuária Brasileira, Brasília, (34): 1517-1527.

Araujo, H. F. P. ; Vieira-Filho, A. H. ; Cavalcanti, T. A. \& BArbosA, M. R. V. 2012. As aves e os ambientes em que elas ocorrem em uma reserva particular no Cariri paraibano, nordeste do Brasil. Rev. Brasileira de Ornitologia. (20): 365-377.

ARgEL-DE-OliveirA, M. M. 1998. Aves que plantam. Frugivoria e dispersão de sementes por aves. Bolm CEO, (13): 9-23.

Borges, L. F. R. SCOlforo, J.R.; OliveirA, A.D.; Mello, J.M.; ACERbi-Junior, F. W. \& FreiTAS, G. D. 2004. Inventário de fragmentos florestais nativos e propostas para seu manejo e o da paisagem. Cerne, (10): 22-38.

Cazetta, E., Rubim, P., Lunardi, V.O., FrancisCo, M.R. \& GAletTI, M., 2002. Frugivoria e dispersão de sementes de Talauma ovata (Magnoliaceae) no sudeste brasileiro. Ararajuba, (10): 199-206.

CBRO - COMITÊ BRASILEIRO DE REGISTROS ORNITOLóGıCOS. 2013. Lista das aves do Brasil. Disponivel em: http://www.cbro.org.br. Acesso em: $12 / 03 / 2013$.

DAJOz, R. 1983. Ecologia Geral. Rio de Janeiro: Editora Vozes, 472 p.

Faustino, T. C. \& MAChado, C. G. 2006. Frugivoria por aves em uma área de campo rupestre na Chapada Diamantina, BA. Ararajuba, São Leopoldo, (14): 137-143.

FonSECA, F. Y. \& ANTUNES, A. Z. 2007. Frugivoria e predação de sementes por aves no Parque Estadual Alberto Löfgren. Revista do Instituto Florestal, (19): 81-91.

Francisco, M. R. \& Galetti, M. 2001. Frugivoria e dispersão de sementes de Rapanea lancifolia (Myrsinaceae) por aves numa área de cerrado do Estado de São Paulo, sudeste do Brasil. Ararajuba, Londrina, (9): 13-19.

Francisco, M. R. \& GalettI, M. 2002.Aves como potenciais dispersoras de sementes de Ocotea pulchella Martius (Lauraceae) numa área de vegetação de cerrado do sudeste brasileiro. Revista Brasileira de Botânica, São Paulo, (25): 11-18.

GondIM, M. J. C. 2001. Dispersão de sementes de Trichilia spp. (Meliaceae) por aves em um fragmento de mata mesófila semidecídua, Rio Claro, SP, Brasil. Ararajuba, Londri- 
na, (9): 101-112.

Guerrero, S.R. \& Figueiredo, R.A. 1997. Influência de uma ave neotropical (Turdus rufiventris Vieillot) sobre a germinação das sementes da figueira-asiática (Ficus microcarpa Linn. f.). Biotemas, Florianópolis, (10): 27-34.

HeRrera, C.M. \& JORDANO, P. 1981. Prunus mahaleb and birds: the high-eficiency seed dispersal system of a temperate fruiting tree. Ecological Monographs, (51): 203-218.

Johnson, R. A. \& WiChern, D.W. 1988. Applied Multivariate Statistical Analysis. Prentice Hall International, Inc. New Jersey, 607p.

JoRdano, P.; GALETTI, M.; PIZO, M.A. \& Silva, W.R. 2006. Ligando frugivoria e dispersão de sementes à biologia da conservação, pp. 411-436. In: (C.F.D. Rocha, H.G. Bergallo \& M.A.S. Alves, eds). Biologia da conservação: essências. Rima, São Carlos.

Kageyama, P. Y.; Gandara, F. B. \& SouzA, L. M. I. 1998. Conseqüências genéticas da fragmentação sobre populações de espécies arbóreas. Série Técnica IPEF, (12): 65-70.

LEVEY, D.J. 1987. Seed size and fruit-handling techniques of avian frugivores. The American Naturalist. (129): 471-485.

MANhÃES, M. A. 2003. Dieta de Traupíneos (Passeriformes, Emberizidae) no Parque Estadual do Ibitipoca, Minas Gerais, Brasil. Ihe- ringia: Série Zoologia, Porto Alegre, (93): 59-73.

MAnhÃES, M. A.; ASSIS, L. C. S.; \& CAStro, R. M. 2003. Frugivoria e dispersão de sementes de Miconia urophylla (Melastomataceae) por aves em um fragmento de Mata Atlântica secundária em Juiz de Fora, Minas Gerais, Brasil. Ararajuba, Seropédica, (11): 173-180.

MARCONDES-MACHAdO, L. O. 2002. Comportamento alimentar de aves em Miconia rubiginosa (Melastomataceae) em fragmento de cerrado, São Paulo. Iheringia: Série Zoologia, Porto Alegre, (92): 97-100.

Marcondes-Machado, L. O. M. \& Rosa, G. A. B. 2005. Frugivoria por aves em Cytharexyllum myrianthum cham (Verbenaceae) em áreas de pastagens de Campinas, SP. Ararajuba, São Leopoldo, (13): 113-115.

MustajarVI, K.; Silkamaki, P.; RYtKonen, S. \& LAMMI, A. 2001. Consequences of plant population size and density for plant: pollinator interactions and plant performance. Journal of Ecology, (89): 80-87.

Nascimento, H. E. M.; Dias, A. S.; Tabanez, A. A. J. \& VIANA, V. M. 1999. Estrutura e dinâmica de populações arbóreas em um fragmento de floresta estacional semidecidual na região de Piracicaba, SP. Revista Brasileira de Biologia, São Carlos, (59): 329-342.

OliveirA, S. L.; Mohr, L. R. S. \& Kohler, A. 2009. Guildas alimentares de aves em ambientes 
degradados no bioma pampa, RS, Brasil. In: CONGRESSO DE ECOLOGIA DO BRASIL, 9, 2009, São Lourenço. Anais... São Lourenço.

PAscotto, M. C. 2006. Avifauna dispersora de sementes de Alchornea glandulosa (Euphorbiaceae) em uma área de mata ciliar no estado de São Paulo. Revista Brasileira de Ornitologia, São Leopoldo, (14): 291-296.

Pizo, A. M. \& VieIRA, E. M. 2004. Granivorous birds as potentially important post: dispersal seed in a Brazilian Forest Fragment. Biotropica, (36): 417-423.

Primack, B.R. \& Rodrigues, E. 2001. Biologia da conservação. Editora Planta, Londrina, $327 p$.

Rabello, A., Ramos, F. N. \& Hasui, E. 2010. Efeito do tamanho do fragmento na dispersão de sementes de Copaíba (Copaifera langsdorffii Delf.). Biota Neotropica, São Paulo, (10): 47-54.

Reis, A.; Zambonin, R. M. \& Nakazono, E. M. 1999. Recuperação de áreas florestais degradadas utilizando a sucessão e as interações planta animal. Série Cadernos da Biosfera. Conselho Nacional da Reserva da Biosfera da Mata Atlântica/Governo do Estado de São Paulo. São Paulo, caderno 14, $42 p$.

RodA, S.A. 2003. Aves do Centro de Endemismo Pernambuco: composição, biogeografia e conservação. Tese de Doutorado. Universidade Federal do Pará, Belém, 520p.
RudGe, A. C. 2008. Contribuição da chuva de sementes na recuperação de áreas e do uso de poleiros como técnica catalisadora da sucessão natural. Dissertação (Mestrado em Conservação da Natureza, Ciências Ambientais e Florestais). Instituto de Florestas, Departamento de Silvicultura, Universidade Federal Rural do Rio de Janeiro, Seropédica, RJ.

SANTOS, T. \& TelleRIA, J. L. 1994. Influence of forest fragmentation on seed consumption and dispersal of spanish juniper (Juniperus thurifera). Biological Conservation, (70): 129-134.

SICK, H. 1997. Ornitologia Brasileira. Rio de Janeiro: Editora Nova Fronteira, 862p.

Silva, J. M. C.; SouzA, M. A.; Bieber, A. G. D. \& CARLOS, C. J. 2003. Aves da Caatinga: status, uso do habitat e sensitividade. In: LEAL, I. R.; TABARELLI, M. \& SILVA, J. M. C. (eds). Ecologia e Conservação da Caatinga. Recife, Editora Universitária da UFPE, 822p.

Stotz, B.F.; FItZPATRICK, J.W.; PARKER III, T.A. \& MoskovitZ, D.K. 1996. Neotropical birds: Ecology and Conservation. Univ. Chicago Press, Chicago, 478p.

VAlente, R. M. 2001. Comportamento alimentar de aves em Alchornea glandulosa (Euphorbiaceae) em Rio Claro, São Paulo. Iheringia: Série Zoologia, Porto Alegre, (91): 1-8.

Willis, E. O. 1979. The composition of avian communities in remanescent woodlots in 
southern Brazil. Papéis Avulsos de Zoologia, São Paulo, (33): 1-25.
Recebido: 01/10/2015

Revisado: 20/04/2016

Aceito: 10/05/2016 
12. FERREIRA et al.

Revista Brasileira de Zoociências 18(2): 1-12. 2017 